was the evaluation of the short-term success and safety after PV stenting of severe stenosis.

Methods and results 5 patients (pts) with 11 PVS were prospectively evaluated. All 5 pts experienced onset of dyspnoea on exertion some weeks or several months before, 3 with dyspnoea at rest, 5 with cough, and 2 with chest pain. On multirow spiral CT examination for each case, the narrowest lumen of the affected PVs measured $2 \pm 1.1 \mathrm{~mm}$ compared with $10 \pm 1.3 \mathrm{~mm}$ at baseline $(p \leqq 0.001)$. The length of narrow segment was $10 \pm 2.9 \mathrm{~mm}$. All of 11 veins were treated with stent implantation with Palmaz Genesis stents, which size were 7- or 8-mm-diameter and 12 -, 15-, 18 -mm-length. At percutaneous intervention, these veins showed $81 \pm 13 \%$ stenosis, with a mean gradient of $20 \pm 5 \mathrm{~mm} \mathrm{Hg}$. This was significantly reduced to a residual stenosis of $8 \pm 2 \%(p \leq 0.001)$ and a residual gradient of $3 \pm 2 \mathrm{~mm} \mathrm{Hg} \quad(p \leq 0.001)$ immediately. The mean pulmonary pressure was $46 \pm 5 \mathrm{~mm} \mathrm{Hg}$ pre-intervention, which decreased to $28 \pm 4 \mathrm{~mm} \mathrm{Hg}$ post-intervention in our protocols. The procedure was safety and successful, no complications were occurred in our pts. The symptomatic response was improved immediately and significantly.

Conclusions Percutaneous intervention produces rapid and dramatic symptom relief in patients with highly symptomatic PV stenosis after radiofrequency ablation for AF. Nevertheless, mid-term and long-term follow-up study with flow determination in the affected vein is still need to assess.

\section{E0490 SAFETY AND FEASIBILITY OF TRANSRADIAL CORONARY ANGIOGRAPHY AT THE OUTPATIENT CLINIC}

doi:10.1136/hrt.2010.208967.490

Yang Shiwei, Zhou Yujie, Hu Dayi, Shi Dongmei, Yang Qing, Wang Jianlong, Cheng Wanjun, Nie Bin, Wang Zhijian, Li Yunzhi, Liu Xiaoli. Beijing Anzhen Hospital Affiliated to Capital Medical University

Objective To evaluate the safety and feasibility of transradial coronary angiography at the outpatient clinic.

Methods From February, 2008 to June, 2008, 100 outpatients who received transradial coronary angiography in Anzhen Hospital were included in this analysis, and 100 inpatients who underwent coronary angiography were selected as control group. Primary endpoints included success rate, percentage of angiographic catheter use with different diameters, adverse events during the procedure (such as death, malignant arrhythmia, acute myocardial infarction, coronary artery spasm, coronary artery dissection, perforation or occlusion, etc.) and after the procedure (such as death, acute myocardial infarction, upper limb haematoma, osteofascial compartment syndrome, radial artery pseudoaneurysm or occlusion, etc.).

Results The success rate $(100 \%$ vs $100 \%)$, procedure duration time $(12.5 \pm 3.4) \mathrm{min}$ vs $(10.8 \pm 3.6) \mathrm{min}, \mathrm{p}=0.517)$ and exposition time $(4.3 \pm 1.0) \mathrm{min}$ vs $(4.1 \pm 1.0) \mathrm{min}, \mathrm{p}=0.629)$ were similar between the outpatient and inpatient groups. Radial and coronary artery spasm were the main adverse events during the angiography, and haematoma was the main adverse event after the angiography. There were no significant differences of adverse events between the 2 groups. the total cost of the outpatient group was significantly lower than the inpatient control group $(4012 \pm 238)$ yuan vs $(5329 \pm 371)$ yuan, $\mathrm{p}<0.01$ ). Expenditure including chemical tests, medicine, nursing care, Room \& Board all decreased significantly.

Conclusion Transradial coronary angiography application at the outpatient clinic was safe and feasible for stable patients, and this procedure could decrease the medical expenditure and shorten the admission time.

\section{E0491 ASSOCIATION OF CORONARY HEART DISEASE WITH CAROTID ARTERY INTIMA-MEDIA THICKNESS AND BAPWV}

doi:10.1136/hrt.2010.208967.491

Liu Junming, Huang Menjun, Wang Mingjian, Xie Wei, Dou Qinghui, Zou Qi. Department of Cardiovascular Internal Medicine, Bingtuan Hospital of Production and Construction, China

Objective To investigate the association of coronary heart disease with carotid artery intima-media thickness and baPWV, by measuring carotid intimia - media thickness and baPWV.

Methods 160 patients who had been examined by coronary angiography were divided into normal group and coronary artery disease group (this group was subdivided into 1 vessel group and multi-vessel group). Carotid ultrasonography and baPWV were performed on them

Results Compared to the patients in normal group, The carotid artery IMT of the coronary artery disease group increased significantly with the aggravation of coronary artery stenesis (the norma group: $0.83 \pm 0.08 \mathrm{~mm}$, the 1 vessel group: $0.91 \pm 0.11 \mathrm{~mm}$, the multivessel group: $1.08 \pm 0.15 \mathrm{~mm}, \mathrm{p}<0.01$ ). The baPWV increased too (the normal group $1411.20 \pm 197.71$, the 1 vessel group $1742 \pm 200.89$, the multi-vessel group $2839.40 \pm 319.03, \mathrm{p}<0.01)$. The carotid artery IMT and baPWV were significantly higher in multi-vessel group than those in 1 vessel group and normal group $(p<0.01)$.

Conclusion Carotid atherosclerosis and baPWV have great value in predicting coronary artery disease. For the coronary heart disease high risk group, routine carotid ultrasound examine and the measure of baPWV can be used to estimate the state of coronary artery atherosclerosis.

\section{e0492 CAROTID INTIMIA-MEDIA THICKNESS AND CORONARY ARTERY DISEASE}

doi:10.1136/hrt.2010.208967.492

Liu Junming, Huang Wenjun, Wang Mingjian, Xie Wei, Dou Qinghui, Zou Oi. Department of Cardiovascular Internal Medicine, Bingtuan Hospital of Production and Construction, Xinjiang, China

Objective To analyse the relationship of carotid atherosclerotic and coronary artery disease (CAD), by measuring Carotid intimia media thickness.

Methods The plaque index and intimia - media thickness (IMT) were detected by high-resolution ultrasound in patients with coronary artery angiography. The extent of difference of IMT were compared between patients with and without coronary artery disease.

Results Compared to the patients with no CAD, the IMT of patients with CAD significantly increased $(0.83 \pm 0.08 \mathrm{~mm}$ vs $0.91 \pm 0.11 \mathrm{~mm} ; 0.83 \pm 0.08 \mathrm{~mm}$ MS1.08 $\pm 0.15 \mathrm{~mm} ; 0.91 \pm 0.11 \mathrm{~mm}$ MS1.08 $\pm 0.15 \mathrm{~mm} ; \mathrm{p}<0.05)$. The incidence of plaque in CAD patients was higher than that of patients with no CAD. The IMT was significantly higher in multi-vessel group than those in univessel group $\mathrm{p}<0.05)$.

Conclusion In CAD patients, the widened inner diameter and lower blood flow rate of carotid artery result in a maximal shearing force significantly lower than that of the control group.The increasing intima-media thickness is associated greatly with carotid atherosclerosis. The haemodynamics change plays an important role in the development and progression of atherosclerosis in CAD patients. Carotid atherosclerosis has great value in predicting coronary artery disease. The IMT can be the surrogate markers of the extensive of coronary artery lesion. 